\title{
Acupressure: A brief review on alternative treatment approach for Polycystic Ovarian Syndrome
}

\author{
Shriyanka Mishra, Himani Nautiyal* and Sanjay Singh \\ Department of Pharmacology, Siddhartha Institute of Pharmacy, Near IT Park, Sahastradhara Road, \\ Dehradun, Uttarakhand, India \\ *Corresponding Author email: shriyankamishra4@gmail.com
}

Received: 24.8.2021; Revised: 20.12.2021; Accepted: 21.12.2021

(O) Society for Himalayan Action Research and Development

\begin{abstract}
Acupuncture is a Traditional Chinese medical practice (TCM); where thin needles are pricked at specific points in the body. In Ancient time, this practice was used to relieve pain but now it has become a therapy to treat other conditions. As an ancient practice, acupressure uses healing power of the body to treat itself by stimulating specific points. Different studies have found positive effects of acupressure in polycystic ovarian syndrome (PCOS), as it has been effectively used to procure regular menstrual frequency and normalize the androgen hormone levels. According to acupressure theory, an energy flow pattern is essential for health, when this energy flow is disturbed in body leads to diseases. These acupressure points are present on meridians and vital energy "qi" flows along it, though no anatomical and histological existence is available for such points. Present review briefly describes these points.
\end{abstract}

Key words - Acupressure, Acupuncture, Traditional Chinese Medicine, PCOS

\section{Introduction-}

Since ancient times, acupressure technique was used to relieve pain such as allergic rhinitis, depression, headache, nausea and vomiting including morning sickness, pain in the epigastria, face, neck, tennis elbow, lower back, etc., but later it has been developed as a complete therapy for getting relieve from many ailments. It is a 3000- year old method of treatment that uses the body's own healing power performed by stimulating predefined points on the body. More than 3 million Americans use acupuncture, but it is even more popular in other countries. In France, for example, one in five people have tried acupuncture. Positive effects of acupuncture on menstrual frequency and androgenic hormone levels have been demonstrated. It is also used effectively in some diseases related to endocrinological disorders such as PCOS (Jedel et al 2011, Horasanl et al 2008).

Acupressure consists of pressing the acupuncture points in an attempt to help the free flow of energy in the channel(s). Acupressure is similar to acupuncture, but the person uses their 
fingers instead of needles to work the point. During a treatment or self-treatment, a person will experience a slight pain when the proper acupuncture point is pressed. Acupressure helps to correct functional imbalances and restore the flow thus returning the body to a more natural state of well-being (Stener-Victorin et al 2019). It is also believed that 12 major meridians connect specific organs or networks of organs, organizing a system of communication throughout the body. The meridians begin at fingertips, connect to brain, and then connect to an organ associated with a certain meridian. According to this theory, when one of these meridians is blocked or out of balance, illness can occur. Acupressure and acupuncture are among the types of TCM that are thought to help restore balance (Stener-Victorin et al op cit).

\section{Acupressure as an Alternative Therapy in PCOS}

Polycystic Ovarian Syndrome (PCOS) is a common endocrine disorder in women of reproductive age. It can affect ability to have a child (fertility). A patient of PCOS produce higher than normal amounts of male hormones. The hormone imbalance causes skip menstrual periods (Azziz et al 2013). PCOS affects women ovaries; it is the reproductive organs which are produce estrogen $\boldsymbol{\&}$ progesterone hormones that regulate menstrual cycle. These ovaries also produce a small amount of male hormones called androgens. In PCOS condition, ovaries secreted large amount of this hormone (androgens) called Hyperandrogenism (Azziz et al op cit). The lack of ovulation alters levels of estrogen, FSH \& LH. Estrogen \& Progesterone levels are lower than normal, while androgen higher than normal. In PCOS many small, fluid-filled sacs grow inside the ovaries.

The word "polycystic" means "many cysts". These sacs are actually follicles, which one containing an immature egg. The eggs never mature enough to trigger ovulation . PCOS also causes hair growth on face $\&$ body, baldness. It can contribute to long term health problems like Diabetes, heart disease, inflammation etc. (Azziz et al op cit). Auricular point's acupressure (AA) may also serve as alternative management for PCOS for its benefits in both physical and psychological well-being (Azziz et al op cit).

The most obvious neuro-endocrine feature in PCOS is increased luteinizing hormone (LH) pulsatility, with relatively low follicular stimulating hormone (FSH) secretion (Johansson, Stener-Victorin 2013). One of the most common features of PCOS is insulin resistance, reported in $62 \%$ to $95 \%$ of women with PCOS (Stepto et al 2013). An increase in androgen level also affects around $60 \%$ to $80 \%$ of women with PCOS. It can produce hirsutism and alopecia (Azziz 2006). Acupuncture has also been reported to potentially improve insulin sensitivity and to decrease testosterone in patients with PCOS (Johansson et al 2013, Kuang et al 2013). 


\section{Acupuncture Nerves, Points and Methods Associated with PCOS-}

Electro acupuncture for PCOS: There are various methods for applying acupuncture. Electroacupuncture is one of the methods of complementary medicine for these patients with no side effect. It can improves ovulation by affecting secretion of beta-endorphins in CNS and inhibition of sympathetic tone. Also EA increases uterine blood flow resulting in reduction of uterine vasoconstriction by sympathetic nervous system (Stener-Victorin et al 1996, StenerVictorin et al 2000, 2009, ). If the sessions of EA will be performed again and again then it results decrease in the testosterone and the ratio of $\mathrm{LH}$ to FSH, and finally improve the menstrual regularity in women with PCOS complaining from amenorrhea or irregular menstruation, with no side effects. (Chen, Yu 1991).

\section{Auricular Points Acupressure for PCOS-}

Another method for acupuncture is Auricular Points Acupressure which is a therapeutic approach to treats various disorders by stimulating specific points on the ear. AA has made rapid progress in the medical practice in China AA applies vaccaria seeds or steel beads on auricular acu points to stimulate cranial nerves/spinal nerve on the auricle of the ear which causes a reaction in the central nervous system.

While working with high-risk diabetes it showed that AA could increase the concentration of antioxidative enzymes to alleviate oxidative stress (Shiraishi et al 1995). Auricular acu points stimulation may increase the secretion of a peptide hormone ghrelin that induces satiation which could also decrease leptin levels that is associated with BMI and IR in PCOS patients (Hsu et.al. 2009).

\section{Effects of Auricular Pressure Combined With Low-Calorie Diet}

The auricular acupressure combined with low calorie diet on leptin hormone has also been considered effective. The absence of leptin leads to uncontrolled food intake, leading to obesity. Acupuncture has been found effective in weight loss and suppression of appetite in some studies (Kang et al 2005).

\section{Electroacupuncture-}

Electroacupuncture is a modified form that uses two needles. A mild electric current passes between these needles during treatment. This current generally applies more stimulation to acupoints than needle twirling or other hand manipulation techniques an acupuncturist might use. 


\section{Points of Spleen Meridian-}

An acupuncture meridian that begins at the great toe, extends up the leg, crosses the front of the body and ends above the armpit. Stimulation of acupoints along the spleen meridian is used for reproductive, urinary and gastrointestinal tract conditions. It originates at the medial corner of the big toe and runs externally along the medial side of the foot. It also emerges to continue externally from a point on the upper abdomen to continue upward on the lateral side of chest.

\section{Points of Stomach Meridian}

The stomach is one of the longest meridians, with 45 points. The Stomach is the Yang partner of the Yin Spleen. The stomach is called the 'Minister of the Mill', the 'Sea of Nourishment', and the 'Root of Postnatal Life', because it is responsible for providing the entire body with energy from the digestion of food and fluids. In addition to digesting foods and liquids and moving them to the small intestine for extraction and assimilation, the stomach also has an important energetic job. It extracts energy from foods and fluids, and coordinates with spleen to transport that energy to the lungs. There it combines with Qi from the air we breathe. Any dysfunction of the stomach creates immediate imbalance in the other organs

\section{Conception Vessel}

These points are very important to acupuncturists because they back up treatments using other acupuncture points. Many of these are vital in developing and influencing Yin energy. Others are powerful local points, for energy, the womb, the stomach and so on. Those little dots up the mid-line of the torso don't really do justice to the path of the Conception Vessel. It starts, so all the ancient texts on the matter say, in the low abdomen, probably in the uterus in women and possibly in the prostate gland in men.

\section{Front-Mu Points}

Front-mu Points $(\mathrm{mu})$ are the points on the chest and abdomen where qi of the respective zangfu organs is infused. They are located on the chest and abdomen in close proximity to their respectively related zangfu organs, hence the name Front-mu points. Each of the zangfu organs and the Sanjiao has the Front-mu point, twelve in all.

\section{Back-Shu Points}

The Back Shu points are located on the inner pathway of the Bladder meridian on both sides of the back. This pathway is located half-way between the spinal column, and the medial (inner) border of the shoulder blade (scapula). The Back Shu points are located on the inner pathway of the Bladder meridian on both sides of the back. This pathway is located half-way between the spinal column, and the medial (inner) border of the shoulder blade (scapula). 


\section{References-}

Azziz R, Carmina E, Chen Z, et al. (2016). Polycystic ovary syndrome. Nat Rev Dis Primers; 2:16057.

Azziz R, Carmina E, Dewailly D, et al. (2006) Positions statement: criteria for defining polycystic ovary syndrome as a predominantly hyperandrogenic syndrome: an Androgen Excess Society guideline. J Clin Endocrinol Metab; 91: 4237-45.

Chen BY, Yu J. (1991) Relationship between blood radio immune-reactive beta-endorphin and hand skin temperature during the electro-acupuncture induction of ovulation. Acupunct Electrother Res.; 16:1-5.

Horasanl E,Usta B, Yesilay A (2008) Medical Acupuncture. 52: 70-75.

Hsu CH, Wang CJ, Hwang KC, et al. (2009). The effect of auricular acupuncture in obese women: a randomized controlled trial. $J$ Womens Health; 18:813-8.

Jedel E, Labrie F, Odén A, Holm G, Nilsson L, et al. (2011) Impact of electro-acupuncture and physical exercise on hyperandrogenism and oligo/amenorrhea in women with polycystic ovary syndrome: a randomized controlled trial. Am J Physiol Endocrinol Metab 300: E37- E45.

Johansson J, Stener-Victorin E. (2013). Polycystic ovary syndrome: effect and mechanisms of acupuncture for ovulation induction. Evid Based Complement Alternat Med $2013 ;: 762615$.

Kang SB, Gao XL, Wang SJ, et al. (2005 ) Acupuncture for treatment of simple obesity and its effect on serum leptin level of the patient. Zhongguo Zhen Jiu; 25:243-5.

Kuang H, Li Y, Wu X, et al. (2013). Acupuncture and clomiphene citrate for live birth in polycystic ovary syndrome: study design of a randomized controlled trial. Evid Based Complement Alternat Med; 2013: 527303.

Liang FKD (2010).Acupuncture: is it effective for treatment of insulin resistance? Diabetes Obes Metab; 12:555-69.

Stener-Victorin E, Jedel E, Janson PO, Sverrisdottir YB (2009). Low-frequency electroacupuncture and physical exercise decrease high muscle sympathetic nerve activity in polycystic ovary syndrome. Am J Physiol RegulIntegr Comp Physiol. ; 297:R387-R395.

Stener-Victorin E, Waldenstrom U, Andersson SA, Wikland M. (1996). Reduction of blood flow impedance in the uterine arteries of infertile women with electro-acupuncture. Hu Reprod. ; 11(6):1314-1317.

Stener-Victorin E, Waldenstrom U, Tagnfors U, Lundeberg T, Lindstedi G, Janson PO (2000). Effects of electro-aclupuncture on anovulation in women with polycystic ovary syndrome. Acta obstet Gynecol Scand ; 79:180-188.

Stener-Victorin E, Zhang H, Li R, et al.(2019). Acupuncture or metformin to improve insulin resistance in women with polycystic ovary syndrome: study protocol of a combined multinational cross sectional case-control study and a randomised controlled trial. BMJ Open ; 9:024733.

Stepto NK, Cassar S, Joham AE, et al. (2013). Women with polycystic ovary syndrome have intrinsic insulin resistance on euglycaemic-hyperinsulaemic clamp. Hum Reprod;28:777-84. 